Rep Reports

\title{
A proximity ligation assay using transiently transfected, epitope-tagged proteins: application for in situ detection of dimerized receptor tyrosine kinases
}

Aaron Gajadhar ${ }^{1}$ and Abhijit Guha ${ }^{2}$

${ }_{1}$ Arthur and Sonia Labatt Brain Tumour Research Centre, Hospital for Sick Children, University of Toronto, Canada, and 2 Division of Neurosurgery, Toronto Western Hospital, University of Toronto, Canada

BioTechniques 48:145-152 (February 2009) doi 10.2144/000113354

Keywords: proximity ligation assay; receptor tyrosine kinase; epidermal growth factor receptor; dimerization; in situ; epitope tagging; protein interaction

Supplementary material for this article is available at www.BioTechniques/article/113354.

The development of small molecule and antibody inhibitors targeting the interaction of receptor tyrosine kinases (RTKs), such as epidermal growth factor receptor (EGFR), is of high pharmacological and biological interest. Unfortunately, conventional biochemical techniques using cell or tissue lysates and co-immunoprecipitation experiments to investigate EGFR dimerization are not always conclusive. Here we describe a series of technical and biological validation experiments demonstrating the utility of a proximity ligation assay (PLA)-based methodology for in situ visualization and quantification of ligand-dependent EGFR receptor dimerization in intact cells. Using the PLA approach combined with a universally applicable epitope tagging strategy, we detected EGFR dimers in cells transiently co-expressing FLAG-tagged and MYC-tagged human EGFRs. Our data strongly suggest that PLA can be used to detect ligand-dependent EGFR dimerization and this signal is generated in a protein interaction-based manner, rather than solely due to proximity of target proteins. This application represents a generalized RTK expression strategy for protein-interaction analysis in a transient expression system where antibody epitopes are not known or not unique enough to discriminate between interaction partners. This assay also holds promise as a general RTK dimerization screening tool in tissue specimens to identify potential dimerization inhibitors with clinical relevance.

\section{Introduction}

Conventional biochemical approaches to assess receptor tyrosine kinase (RTK) dimerization in vitro rely on immunoprecipitation (IP) and chemical crosslinking techniques. However, these strategies are not particularly sensitive, do not preserve physiological or cellular compartmentalization, and are not easily amenable to highthroughput screening. Moreover, the lack of suitable antibodies toward the most common RTK deletion and point mutants precludes detailed dimerization analysis by traditional biochemical methods.

The epidermal growth factor receptor (EGFR) is a prototypical member of the ErbB family of RTKs and is aberrantly overexpressed or activated in numerous human cancers (1). Receptor activation is mediated by ligand binding to the extracellular domain, leading to intramolecular conformational rearrangements that facil- itate homo- or heterodimerization with ErbB family members. Dimerization promotes intrinsic kinase domain activation, resulting in phosphorylation on specific tyrosine residues within the cytoplasmic tail and subsequent stimulation of intracellular signaling pathways $(2,3)$. Tumors harboring overexpressed EGFR and ErbB members have the potential for oncogenic signaling and deregulated cellular behavior. In recent years, therapeutic focus has shifted to abrogating aberrant EGFR function at the critical event of dimerization. Clearly, there exists an urgent need for adequate methodologies to evaluate EGFR dimerization from a biological and therapeutic perspective.

In the present report, we describe our work developing and standardizing an application of proximity ligation strategy $(4,5)$ to allow EGFR homo- and heterodimer detection and quantification in a transient expression system. Furthermore, we utilized this system to confirm that dimerization signal is generated in a protein interactionbased manner, rather than solely due to proximity of target proteins. Additionally, this is to our knowledge the first application of in situ PLA in a transient expression system to detect protein interactions.

\section{Methods and materials}

Expression constructs

Human wild-type EGFR-encoding cDNA (lacking stop codon) was PCR-amplified from the pGEM7zf(-):EGFRwt (Promega, Madison, WI, USA) shuttle vector (primer sequences available in Supplementary Table S1) and cloned into pCR2.1-TOPO using the TOPO-TA system (Invitrogen, Burlington, ON, Canada) for sequence verification. EGFRwt-pCR2.1-TOPO was SacII-digested and blunt-end-cloned into the HindIII site of pLPCX expression vector (Clontech, Mountain View, CA, USA) to 

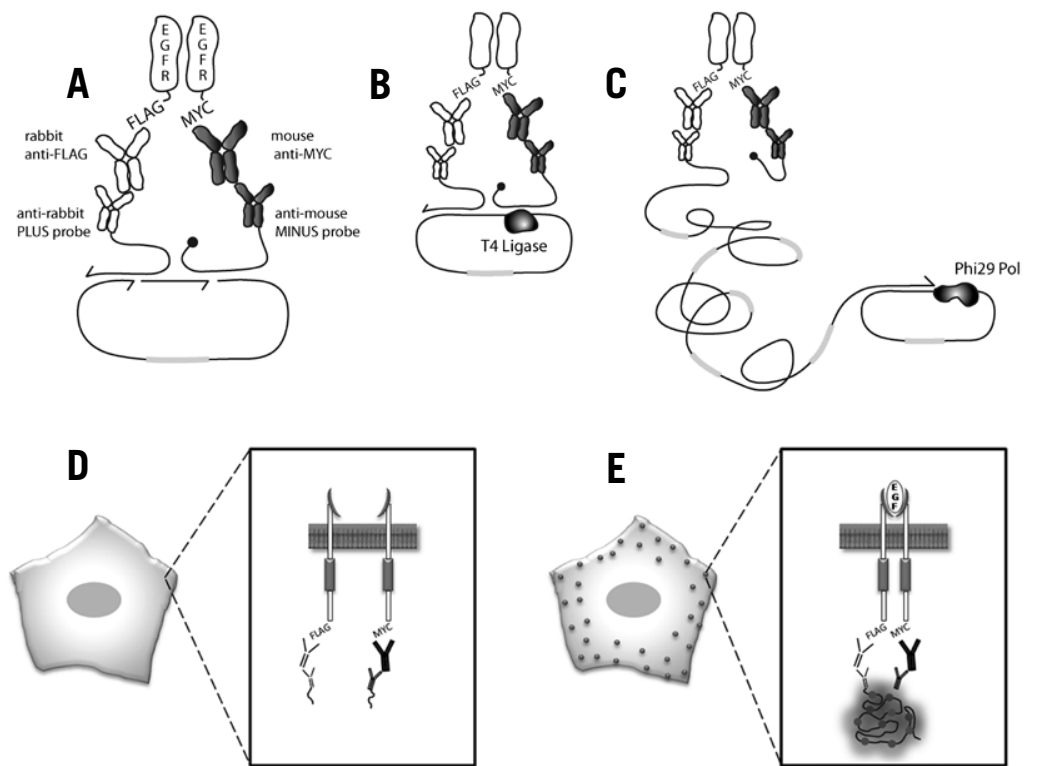

Figure 1. Schematic of epitope-tagging in situ proximity ligation assay. (A) Dual binding of primary antibodies to epitope tags followed by species-selective PLA probe binding provides a scaffold to allow hybridization of connector oligos. (B) T4 DNA ligase catalyzes the ligation of connector oligos to form a continuous circular DNA template. (C) The PLUS proximity probe primes Phi29 polymerasemediated RCA, creating a concatemeric amplification product (the oligo tail of the MINUS proximity probe contains terminal RNA nucleotides, preventing its use as a RCA primer). Hybridization of complementary Texas red-labeled probes to the RCA product (gray area) generates PLA signal. (D) Cells co-transfected with EGFR-FLAG and EGFR-MYC fail to dimerize in the absence of EGF stimulation. (E) Ligand stimulation mediates EGFR construct dimerization, leading to amplification of PLA signal. Each fluorescently labeled bundle represents the formation of one molecular interaction, facilitating the visualization and quantitation of in situ EGFR dimer formation in individual cells.

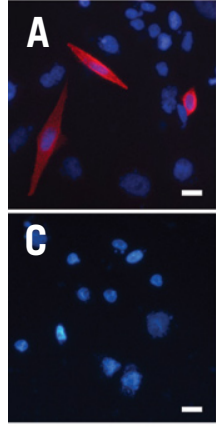

Anti-FLAG IF
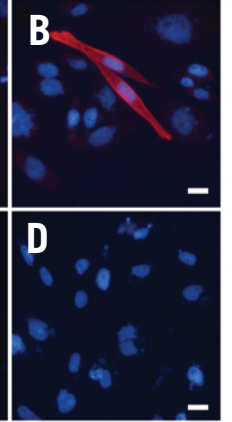

Anti-MYC IF

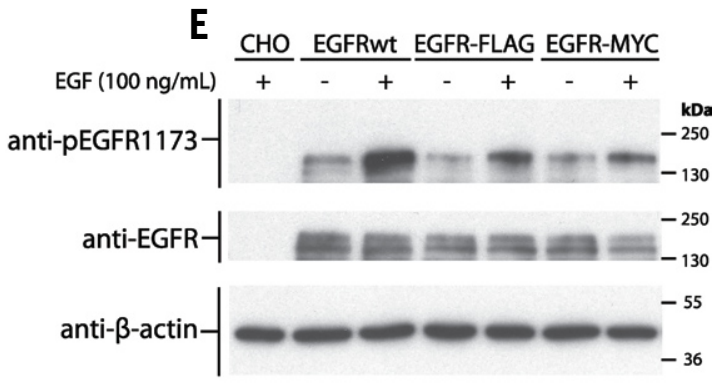

Figure 2. Immunofluorescence and Western blot studies of epitope-tagged EGFR constructs. Localization of EGFR constructs by immunofluorescent staining of CHOK1 cells transfected with EGFR-FLAG (A) and EGFR-MYC (B), as detected with anti-FLAG and anti-MYC antibodies, respectively. Empty vector transfections served as negative controls. ( $C$ and $D$ ) Cells were counterstained with Hoechst 33258 (blue) to visualize nuclei; scale bars $=20 \mu \mathrm{m}$. (E) Immunoblotting to detect phosphotyrosine activation on EGFR-Y1173 of CHOK1 cells transfected with untagged EGFR (EGFRwt; lanes 2 and 3) or MYC- and FLAG epitope-tagged EGFR constructs before and after EGF stimulation.

generate EGFR-pLPCX. To prepare FLAG and MYC epitope tags, oligonucleotides (Integrated DNA Technologies, Coralville, IA, USA) FLAG sense/antisense and MYC sense/antisense (Supplementary Table S1), respectively, were annealed, phosphorylated, and blunt-end-cloned into blunted EcoRI site of EGFR-pLPCX to generate EGFR-FLAG-pLPCX and EGFR-MYC-pLPCX. Untagged EGFR was expressed from a pcDNA3.1/Zeo
(+) construct (Invitrogen). Mammalian expression constructs encoding MYC tag translational fusions of fibroblast growth factor receptor-1 (FGFR1) and ephrin receptor B1 (EPHB1) were generated from hORFeome v5.1 cDNA shuttled into pcDNA3.2/myc-DEST using Gateway (Invitrogen) cloning technology (SIDNET, The Hospital for Sick Children). Y246D point mutant construct was generated using EGFRwt-FLAG-pLPCX as template and mutagenized with a site-directed mutagenesis kit (Stratagene, La Jolla, CA, USA). Nucleotide sequencing was performed to confirm the mutagenesis.

\section{Cell culture and transfection}

Chinese hamster ovary (CHOK1) cells were cultured in $1 \times \mathrm{F}-12 \mathrm{~K}$ nutrient medium containing $10 \% \mathrm{FCS}, 100 \mathrm{U} / \mathrm{mL}$ penicillin, $100 \mu \mathrm{g} / \mathrm{mL}$ streptomycin, and $250 \mathrm{ng} / \mathrm{mL}$ amphotericin B (Wisent, St-Bruno, Quebec, Canada). For immunofluorescence experiments, cells were grown to $70 \%$ confluence on $18 \times 18$-mm glass coverslips (Fisher Scientific, Ottawa, ON, Canada) placed in six-well plates (BD Biosciences, Mississauga, ON, Canada) and transfected with indicated expression constructs using Fugene HD (Roche, Mississauga, ON, Canada), according to the manufacturer's instructions. After $6 \mathrm{~h}$, the transfection medium was changed to serumfree medium. Cells were incubated another 18 $h$ and subsequently washed twice in ice-cold PBS and fixed for 10 min with $4 \%$ paraformaldehyde in PBS at room temperature. For immunoblotting experiments, cells were grown to $70 \%$ confluence in six-well plates and transfected as in immunofluorescence experiments, and serum-starved cells were stimulated with $100 \mathrm{ng} / \mathrm{mLEGF}$ (Chemicon; Billerica, MA, USA).

\section{SDS-PAGE/immunoblotting}

At $24 \mathrm{~h}$ post-transfection, cells were stimulated with EGF as in immunoblotting experiments, rinsed twice in ice-cold PBS, lysed in ice-cold lysis buffer (50 mM HEPES, 150 $\mathrm{mM} \mathrm{NaCl}, 1 \mathrm{mM}$ EGTA, 10\% glycerol (v/v), $1 \%$ Triton X-100 (v/v), 1 mM EDTA, and $10 \mathrm{mM} \mathrm{Na}_{4} \mathrm{P}_{2} \mathrm{O}_{7}$ ), and supplemented with protease inhibitor (Sigma-Aldrich, Oakville, ON, Canada) and phosphatase inhibitor (Calbiochem, Gibbstown, NJ, USA) cocktails. Proteins were resolved on 10\% SDS-polyacrylamide gel and transferred to a PVDF membrane (Perkin Elmer, Woodbridge, ON, Canada). Membranes were immunoblotted with anti-pEGFR1173 antibodies at 1:1000 (Cell Signaling, Danvers, MA, USA), washed, and subsequently incubated with secondary HRP-conjugated antibodies at 1:2500 (Bio-Rad, Hercules, CA, USA), and electrochemiluminescent (ECL) signal was detected with Western Lightning Plus (PerkinElmer, Waltham, MA, USA). Membranes were then stripped and reblotted with 1:1000 anti-EGFR (Upstate, Billerica, MA, USA) and 1:10,000 anti- $\beta$-actin antibodies (Sigma-Aldrich) as described above.

\section{Immunofluorescence}

Fixed cells were delimited with ImmEdge Pen (Vector Laboratories, Burlington, ON, 

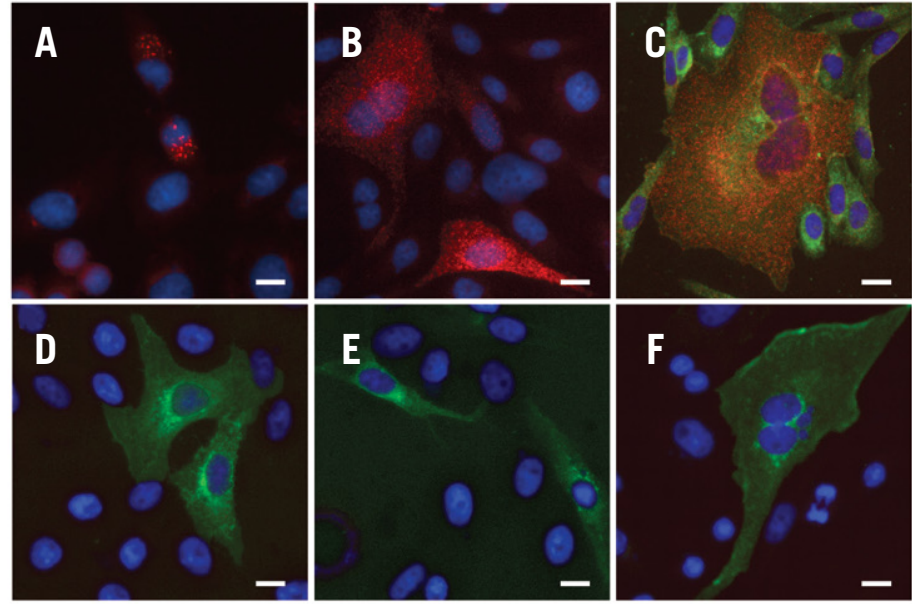

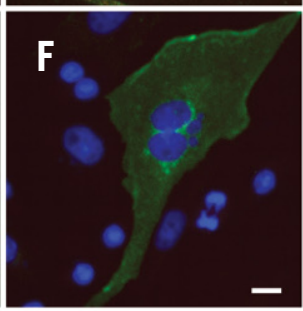

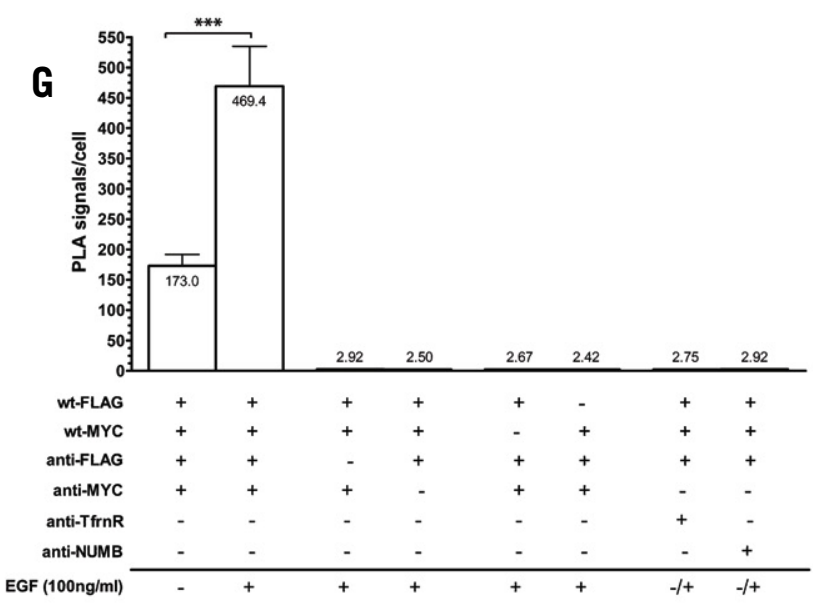

Figure 3. In situ PLA of CHOK1 cells transiently transfected with epitope-tagged EGFR constructs. Prior to EGF stimulation, sparse PLA signal (red) could be detected in CHOK1 cells transiently expressing FLAG and MYC-epitope-tagged EGFR constructs using epifluorescence microscopy (A). However, cells with sharply increased PLA signal could be detected 3 min after $100 \mathrm{ng} / \mathrm{mL}$ EGF stimulation (B). Samples co-stained with anti- $\beta$-actin (C, green signal) allow visualization of cell borders. Negative controls (D-F) show merge of PLA signal (red) with anti-EGFR staining (green). Samples lacking one antibody from the primary pair (D) or one of the EGFR interaction constructs (E) from co-transfection had no detectable PLA signal. Similarly, spurious EGFR interaction with other plasma membrane proteins such as transferrin receptor (F) or Numb (image not shown) was not evident. Scale bars $=20$ $\mu \mathrm{m}$. Quantitation of PLA signal was performed on 30 transfected cells per condition in three independent experiments using semi-automated image analysis; mean PLA signal/cell \pm SEM are plotted (G). There was a statistically significant increase $(* * * P<0.0001)$ after EGF stimulation, with PLA signal dependent on the presence of both anti-FLAG and anti-MYC antibodies and the presence of both partners of the interaction unit. Membrane localization was not sufficient to generate signal as demonstrated by lack of PLA signal using antibodies against TfrnR or Numb.

Canada), permeabilized with $0.1 \%$ Triton $\mathrm{X}-100$ in PBS for 3 min, blocked in $8 \%$ BSA (Calbiochem) for $30 \mathrm{~min}$ at $37^{\circ} \mathrm{C}$, washed, and incubated overnight at $4^{\circ} \mathrm{C}$ in anti-MYC (1:200; Sigma-Aldrich), anti-FLAG (1:500; Sigma-Aldrich), or anti-EGFR (1:500; Upstate) antibodies as indicated. FITC anti-mouse (Abcam,
Cambridge, MA, USA) or Texas red antimouse (Abcam) secondary antibodies at 1:500 were added to washed samples, as indicated, and incubated $1 \mathrm{~h}$ at $37^{\circ} \mathrm{C}$. Cells

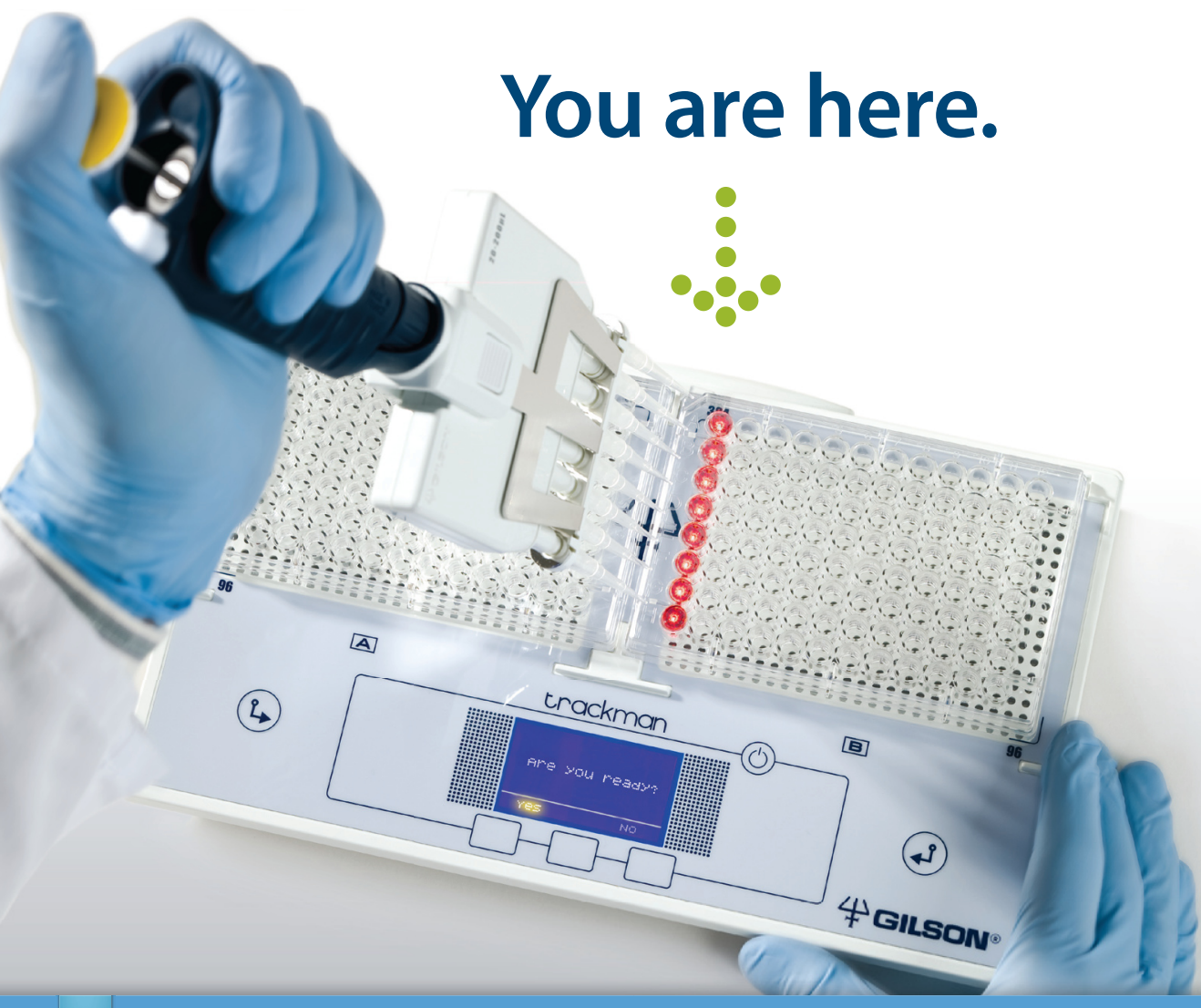

\section{Introducing trackman"}

Get on track, keep on track, as you pipette flawlessly with the help of your new personal laboratory assistant. You'll get greater productivity and eliminate the errors.

- Track your pipetting from two plates, not just one

- Use 96 or 384 well microplates, and single or multichannel pipettes

- 16 programmable possibilities

- Timer function for hands-free operation

- Portable slim design

- Optional tube holder accessory accommodates 2.0, 1.5 and $0.5 \mathrm{~mL}$ tubes

Visit pipetman.com/trackman to learn more. 
were counterstained with Hoechst 33258 and mounted on microscope slides using Vectashield (Vector Laboratories). Images were acquired with a Zeiss Axiovert 200 $\mathrm{M}$ epifluorescence microscope (Carl Zeiss Canada Ltd., Toronto, ON, Canada) under a $20 \times$ objective.

\section{In situ PLA analyses in cultured CHOKI cells}

Cells were grown on coverslips and co-transfected with EGFR-FLAG and EGFR-MYC as described in the "Cell culture and transfection" section. Transfected cells were stimulated with $100 \mathrm{ng} / \mathrm{mL}$ EGF for 3 min, washed with ice-cold PBS, fixed, and permeabilized as described in the "Cell culture and transfection" section. Samples were blocked in $1 \times$ blocking stock (Olink Bioscience, Uppsala, Sweden) for $30 \mathrm{~min}$ at $37^{\circ} \mathrm{C}$ in a humidified chamber. Mouse anti-MYC (1:1000; Sigma-Aldrich) and rabbit anti-FLAG (1:200; Sigma-Aldrich) primary antibodies were diluted in $1 \times$ antibody diluent stock (Olink Bioscience) and co-incubated overnight at $4^{\circ} \mathrm{C}$. Other antibodies used for in situ proximity ligation analysis included anti-transferrin receptor (anti-TfrnR) (Zymed Laboratories, San Francisco, CA, USA), and anti-Numb (provided by Jane McGlade, Hospital for Sick Children, Toronto, Canada).In situ proximity ligation was performed following primary antibody incubation (6). PLA probe dilution/ incubation time and rolling circle amplification (RCA) times were all optimized for this specific application. Anti-mouse PLA MINUS and anti-rabbit PLA PLUS secondary probes (Olink Bioscience) were diluted $1: 5$ in $1 \times$ antibody diluent stock and co-incubated for $45 \mathrm{~min}$ at $37^{\circ} \mathrm{C}$ in a humidified chamber. Oligonucleotides complementary to the proximity probe DNA extensions were incubated as $1 \times$ hybridization stock (Olink Bioscience) and incubated for $15 \mathrm{~min}$ at $37^{\circ} \mathrm{C}$ in a humidified chamber. To complete the rollingcircle priming template, T4 DNA ligase (1:40) was added to samples in $1 \times$ ligation stock (Olink Bioscience), and samples were incubated $15 \mathrm{~min}$ at $37^{\circ} \mathrm{C}$ in a humidified chamber. RCA of the ligated oligonucleotide template was initiated by addition of Phi29 DNA polymerase (1:80) in 1× amplification stock (Olink Bioscience) and incubation for $60 \mathrm{~min}$ at $37^{\circ} \mathrm{C}$ in a humidified chamber. Finally, Texas red-labeled oligonucleotide detection probes (Olink Bioscience) were incubated as $1 \times$ detection stock and incubated for $60 \mathrm{~min}$ at $37^{\circ} \mathrm{C}$ in a humidified chamber. All washing procedures and washing solutions have been described previously (6). FITC anti-actin
(1:100; Abcam) or FITC anti-EGFR (1:50; Upstate), as indicated, were added during the detection step of the PLA reaction. Coverslip samples were mounted with Vectashield and examined with a Zeiss Axiovert $200 \mathrm{M}$ epifluorescence microscope under a $40 \times$ objective.

\section{Quantitative PLA image analysis}

Images were collected using AxioVision acquisition software from several fields of view per condition per experiment. Typically, 30 cells per condition were quantified using Blobfinder 3.1 (Centre for Image Analysis, Uppsala University, Uppsala, Sweden) semi-automated image analysis. Transfected cells were identified with a FITC anti-EGFR stain. Blobfinder analysis involved delineating cells using the signal from one focal plane of Hoechst nuclear stain and enhancing point-like PLA signals to define true signal as local intensity maximum above a background threshold. The number of PLA signals per cell was counted from three Z-plane images. Statistical analysis was performed using GraphPad Prism 4.0 software. Statistical significance was accepted when $P<0.05$ using unpaired two-tailed Student $t$-test. Mean values \pm SEM are presented from quantitation of at least three independent experiments.

\section{Resullts and discussion}

\section{Assay design.}

To circumvent the inherent limitations of other EGFR dimerization detection assays, we developed an in situ dimerization assay by combining epitope tagging with the proximity ligation assay (PLA). In our approach, two different EGFR monomers, tagged at the carboxy terminus with either a FLAG or MYC epitope tag, are co-expressed in a $\mathrm{CHOK} 1$ cell line, which has no endogenous EGFR expression. The overexpressed EGFR-FLAG and EGFR-MYC fusion proteins are probed first with rabbit anti-FLAG and mouse anti-MYC primary antibodies (Figure $1 \mathrm{~A})$, and then with anti-mouse IgG and anti-rabbit IgG that are conjugated to oligonucleotide extensions (6) (Figure 1A). As in standard PLA, the oligonucleotides serve as proximity probes, and if at the requisite distance, hybridize with subsequently added connector oligos to guide the formation of a circular DNA template (Figure 1A). This circular DNA molecule is ligated, primed by one of the proximity probes, and subsequently amplified by RCA to create a single-stranded DNA product covalently attached to one of the proximity probes (Figure 1, B and C). We predicted that prior to EGF stimulation, EGFR monomers would not be close enough to produce PLA signal (Figure 1D), but that ligand-induced EGFR dimerization would enable proximity ligation and subsequent detection by hybridization of Texas red-labeled oligonucleotide probes (Figure 1E). Furthermore, each fluorescently labeled bundle would represent the formation of one molecular interaction, thus facilitating the visualization and quantitation of in situ EGFR dimer formation in individual cells.
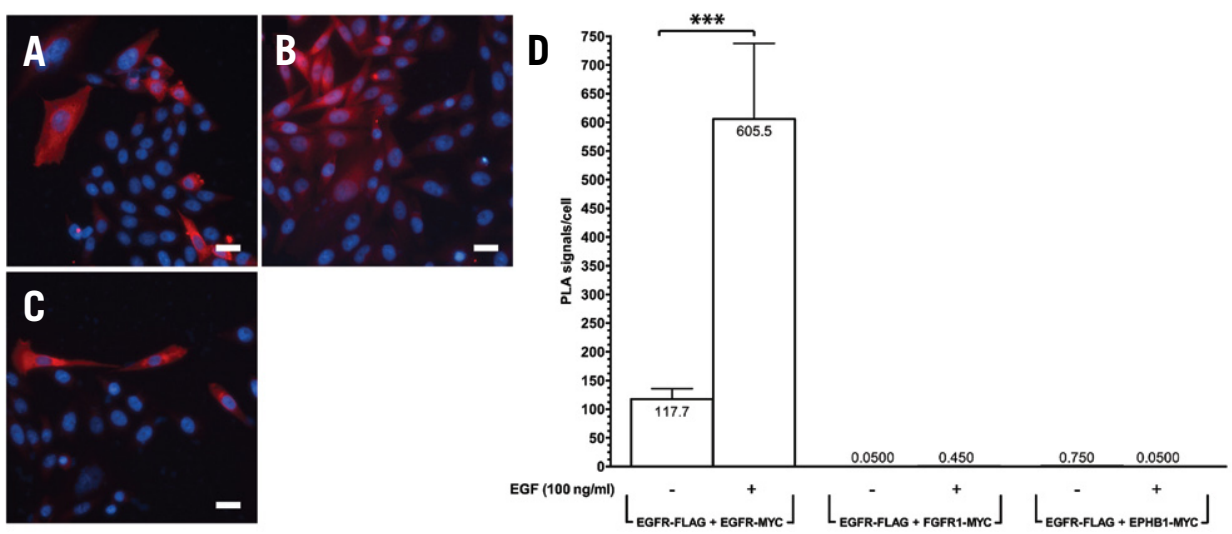

Figure 4. In situ PLA of EGFR-MYC with epitope-tagged FGFR1 or Ephrin receptor B1. Myc-tagged receptor tyrosine kinase (RTK) constructs FGFR1 and EPHB1 show expression levels and transfection efficiency similar to EGFR-MYC constructs (A) in CHOK1 cells by anti-MYC immunofluorescence (B and C); cells were counterstained with Hoechst 33258 (blue) to visualize nuclei. Bars, $20 \mu \mathrm{m}$. As a control for spurious interactions, in situ PLA quantitation of overexpression of EGFR with either FGFR1 or EPHB1 was performed using anti-FLAG and anti-MYC primary antibodies on untreated or EGF-treated samples. Sample images were collected and enumeration was performed on 30 transfected cells per condition in three independent experiments using semi-automated image analysis; mean PLA signal/cell \pm SEM are shown (D). A significant elevation (***P<0.0001) of PLA signal was observed only in cells co-expressing both EGFR-FLAG and EGFR-MYC; there was no detectable signal for cells co-expressing EGFR-FLAG with FGFR1 or EPHB1. 
Activity of epitope-tagged

EGFR fusion constructs

Prior to their use in proximity ligation experiments, we characterized the EGFR-FLAG- and EGFR-MYC-tagged receptors. Immunofluorescence staining of the epitope-tagged receptors in transfected CHOK 1 cells confirmed that both EGFR-FLAG and EGFR-MYC localized to the plasma membrane in an unperturbed manner (Figure 2, A and B).

The carboxyl terminus of EGFR contains the domain that undergoes tyrosine phosphorylation in response to EGF, ultimately allowing recruitment of adaptor proteins and signal transduction propagation. Tyrosine activation occurs on five major phosphotyrosine residues in the distal carboxl terminus of EGFR, although the favored site of autophosphorylation appears to be Tyr-1173 (7). To investigate whether adding an epitope tag near this critical site affected ligandmediated tyrosine phosphorylation, we performed phosphotyrosine immunoblotting. CHOK1 cells transfected with untagged EGFR, EGFR-FLAG, or EGFR-MYC were EGF-stimulated $(100 \mathrm{ng} / \mathrm{mL})$, lysed, and analyzed by Western blot. Similar to untagged
EGFR, EGFR-MYC and EGFR-FLAG demonstrated appropriate ligand-induced phosphorylation (Figure 2E).

In situ PLA detection and quantitation of EGFR dimerization in single cells

Next, we examined cells transfected with EGFR-FLAG and EGFR-MYC expression constructs for ligand-induced PLA signal. There was a substantial increase in PLA signal in EGF-stimulated cells compared with unstimulated control cells (Figure 3, A and B). Although limited transfection efficiency meant only a minority of cells expressed the EGFR fusions, the densely packed PLA signal seen in Figure $3 \mathrm{~B}$ is representative of PLA-positive cells. Proximity probe dilution/incubation time and RCA times were all optimized (data not shown) to ensure detected events did not reach saturation. Co-staining with actin during the detection step shows the EGFR PLA signal was present at the cell periphery (Figure 3C).

To demonstrate the dual recognition requirement for in situ PLA signal generation, use of one primary antibody (for EGFR-MYC or EGFR-FLAG) led to negligible a mounts of PLA signal in EGF-stimulated cells (Figure 3D). Likewise, omission of one of the EGFR epitope-tag constructs from transfection resulted in negligible PLA signal, suggesting the dependence on EGFR recognition for PLA signal (Figure 3E). To test whether spurious PLA signal might be generated simply by shared membrane localization, we substituted the anti-MYC antibody with antibody to transferrin receptor, another endogenous plasma membrane target. There was no PLA signal observed (Figure 3F), indicating that shared membrane localization (Supplementary Figure S1) alone did not suffice to give a PLA signal.

For quantitative analysis of PLA signal, cells were co-stained with FITC anti-EGFR antibody, and EGFR expression-positive cells were selected for analysis by Blobfinder 3.1 software (Centre for Image Analysis). This image-based readout facilitated objective, accurate, and statistically robust quantification of PLA signal. With analysis of only 30 transfected cells per experiment, a 2.71 \pm 0.38 -fold increase in PLA signal/cell was detected after EGF stimulation of the transfected CHOK1 cells $(P=0.0001)$ (Figure $3 \mathrm{G})$. Specificity of the assay was shown by lack of observable PLA signal in negative control transfections or after substitution of one of the EGFR constructs with unrelated

\section{BREAKTHROUGH IN RNA ISOLATION The single step method without phase separation}
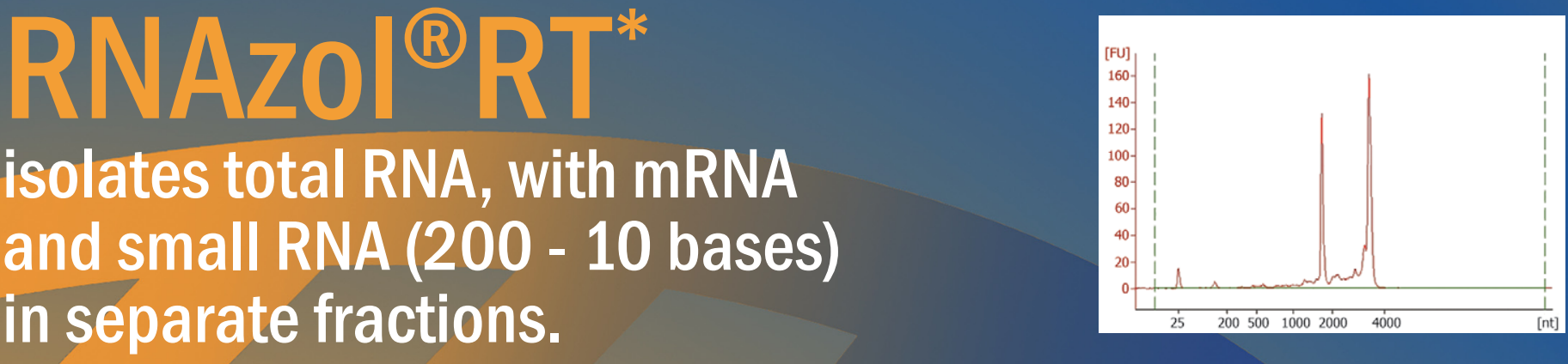

- Higher RNA yield and quality than with previous single-step reagents.

- No chloroform-induced phase separation. Just add water.

- RNA is ready for RT-PCR, microarrays, poly A+ selection, northern blotting

and RNase protection.

- No DNase treatment necessary.

- No need for a refrigerated centrifuge. All steps performed at room temperature.

MOLECULAR RESEARCH CENTER, INC. 5645 Montgomery Road, Cincinnati, Ohio 45212

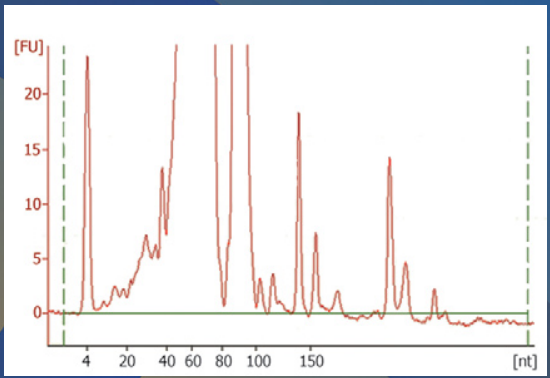



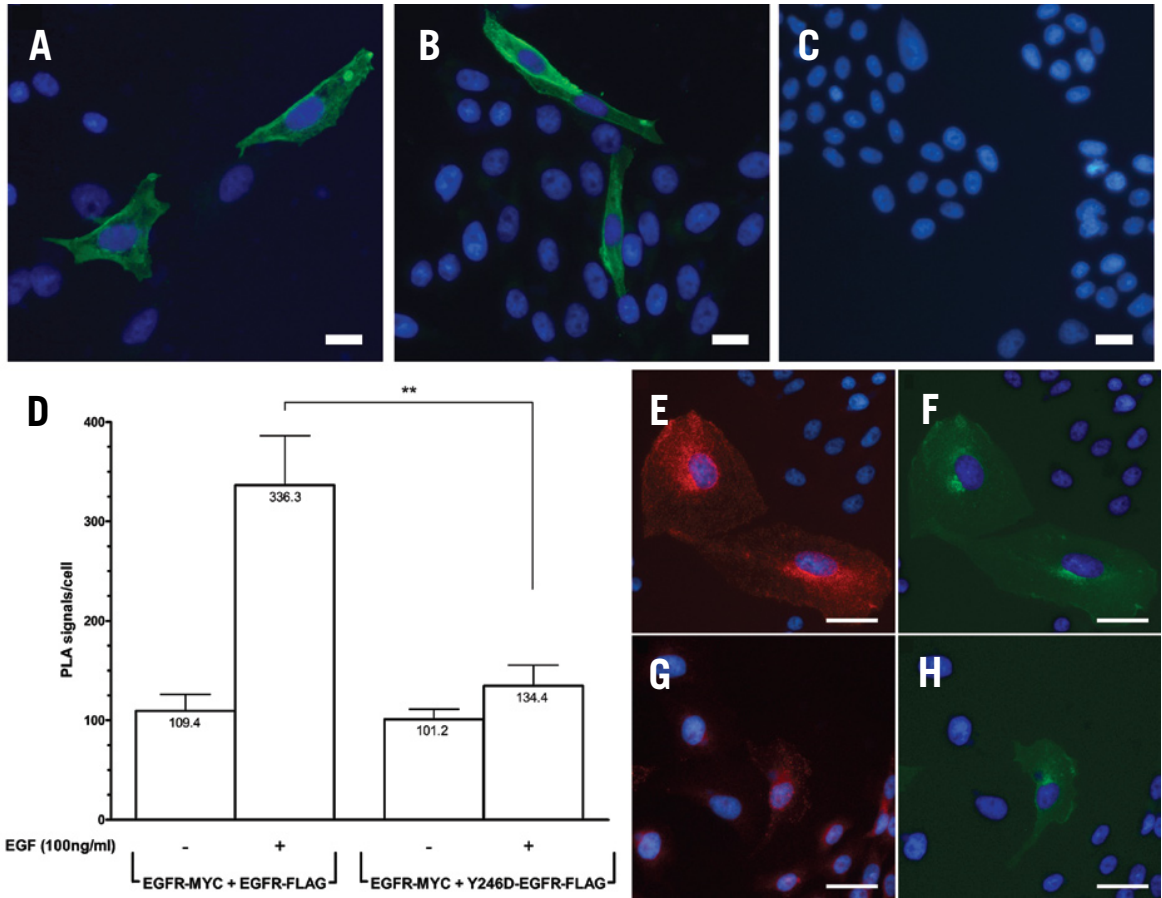

Figure 5. PLA of EGFR dimerization mutant. Compared with the EGFR-FLAG construct (A), anti-EGFR immunofluorescence of EGFR dimerization mutant Y246D shows unaltered localization (B); cells were counterstained with Hoechst 33258 (blue) to visualize nuclei, and untransfected CHOK1 control is shown for comparison (C). Compared with cells transfected with unmutated MYC/FLAG-EGFR, there was a statistically significant decrease in the mean PLA signal/cell from CHOK1 cells transfected with EGFR-MYC and Y246D-EGFR-FLAG (D; ${ }^{*} P=0.0014$; error bars represent SEM). Representative images of in situ PLA signal corresponding to cells transfected with EGFR-MYC/FLAG (E) and Y246D-EGFR-FLAG/EGFR-MYC (G; red signal). Transfected cells in the same field of view were visualized with anti-EGFR stain ( $\mathrm{F}$ and $\mathrm{H}$; green signal). Scale bars $=20 \mu \mathrm{m}$.

membrane proteins such as transferrin receptor or Numb.

As an additional test for spurious PLA signal resulting from non-physiologically relevant co-proximity of overexpressed membrane proteins, mammalian expression constructs encoding carboxy terminal MYC-tagged fibroblast growth factor receptor-1 and ephrin receptor B1 were transiently co-transfected with EGFR-FLAG in CHOK1 cells. In preliminary tests with singly transfected cells stained with anti-MYC antibody, FGFR1-MYC and EPHB1-MYC showed expression levels and membrane localization similar to EGFR-MYC (Figure 4, A-C). However, CHOK1 cells transiently co-overexpressing EGFR-FLAG with FGFR1-MYC or EPHB1-MYC did not generate a detectable PLA signal (Figure 4D).

\section{EGFR dimerization competence} and PLA signal

While the preceding experiments are consistent with enhanced PLA signal as a surrogate of EGFR dimerization, they do not directly demonstrate that assay is dimerization-dependent. To test this, we made an EGFR mutant in which the tyrosine at amino acid 246 is replaced with aspartate. Crystal structures of dimeric extracellular EGFR complexed to ligand suggest that Tyr-246 in the extracellular domain is critical for intramolecular dimerization between two EGFR monomers after ligand binding (8). Mutagenesis studies confirm that alterations to Tyr-246 can severely impair the positioning and stabilization of ligand-dependent EGFR dimerization (9). Anti-EGFR immunofluorescence in CHOK1 cells confirmed that localization of the Y246D EGFR-FLAG mutant (Figure 5B) was unaltered from EGFR-FLAG (Figure 5A) Quantitative in situ PLA was performed using anti-FLAG and anti-MYC antibodies on CHOK1 cells co-transfected with EGFR-MYC and Y246D-EGFR-FLAG (Figure 5D). There was an absence of ligand-induced increase in the PLA signal/cell with EGFR-MYC + Y246D-EGFR-FLAG compared with EGFR-MYC + EGFR-FLAG. Representative images of PLA signal from cells transfected with EGFR-MYC + EGFR-FLAG or EGFR-MYC + Y246D-EGFR-FLAG are shown in Figure 5 (compare $\mathrm{E}$ and G). These results are likely a direct conse- quence of impaired ligand-dependent EGFR dimerization, since both EGFR and Y246D-EGFR samples expressed equivalent levels of EGFR (Figure 5, F and H, respectively), as assessed by staining with FITC-conjugated anti-EGFR. Collectively these data strongly support that the increase in PLA signal reflects ligandinduced EGFR dimerization.

Combining proximity ligation and an epitope-tagging expression strategy, we have devised an approach to visualize and quantify EGFR dimerization in individual cells. This method also represents a generalized RTK expression strategy for protein interaction analysis in a transient expression system where antibody epitopes are not known or not unique enough to discriminate between interacting partners. Importantly, the inclusion of epitope tags to EGFR did not impair the phosphorylation or localization of the receptor. However, further validation of transfection-related effects may be investigated to ensure overexpression does not substantially alter the proteome of transfected cells.

The original in situ PLA for proteinprotein interaction analysis demonstrated the specificity for detecting protein interactions in a particular subcellular region (i.e., cell membrane versus nuclear) (5), but did not investigate the specificity for measuring specific interactions within a particular compartment. The experiments performed in this study strongly suggest that the increase in PLA signal reflects ligand-dependent dimerization of EGFR monomers, as opposed to general proximity of targets: (i) no PLA signal could be detected from EGFR and co-localizing membrane proteins; (ii) overexpression of non-EGFR-interacting RTKs with EGFR did not cause any increase in observed PLA signal between these RTKs and EGFR; and (iii) an EGFR mutant previously shown to be impaired in dimerization ability showed no increase in PLA signal after ligand addition.

One interesting observation was that basal PLA signal was evident in the absence of EGF stimulation. Although the concentrations of the tagged proteins were not sufficient to induce co-proximity of the non-interacting proteins (as seen by the experiments with transferrin, Numb, FGFR, and ephrin receptor B1), it is possible the expression levels may have forced non-physiologically relevant clustering or dimerization of EGFR. However, flow cytometry experiments suggested that the average transfected cell contained $\sim 10^{5}$ EGFRs per cell, which is only $\sim 10$ times more than the physio- 
logical expression level (data not shown). Moreover, our data are consistent with the findings of other studies in which preformed cell surface ligand-independent EGFR dimers were observed and ligand binding was the critical event necessary to induce highaffinity EGFR dimerization and subsequent receptor activation (10-12). The observation that similar levels of EGFR dimerization were observed in samples tested with Y246D-EGFR dimerizationincompetent constructs suggests that basal ligand-independent EGFR dimerization events are mediated by residues of the extracellular domain independent of those implicated in ligand-mediated EGFR intramolecular interactions.

Our epitope-tagging PLA approach joins an array of existing protein-interaction analysis strategies. The workhorse of proteininteraction biology has been co-immunoprecipitants followed by Western blotting analysis. However, this approach is restricted in several ways. This technique may not preserve transient or weak interactions, may require large amounts of cultured cells that may be difficult to obtain in certain cases, and the act of cell lysis does not preserve the subcellular environment in which certain interactions occur. The last decade has also seen a surge in the number of interaction techniques allowing live-cell analysis of protein interactions. Among these are Förster resonance energy transfer (FRET)-based methods involving the fusion of fluorescent proteins, such as cyan fluorescent protein (CFP) and yellow fluorescent protein (YFP), to interacting proteins of interest (13). In this setup, interacting proteins bring CFP donor and YFP acceptor tags into proximity to facilitate the resonance energy transfer upon monochromatic excitation of the acceptor CFP tag. By monitoring dual wavelength emissions from fluorescent tags, one can get a measure of protein interaction ability. Bioluminescent resonance energy transfer (BRET) is a modification of FRET where the CFP fusion is replaced by a chemiluminescent luciferase circumventing the requirement for laser excitation (14). In protein complementation assays (PCA), the protein partners of interest are co-expressed as fusion proteins, each with one portion of a reporter enzyme, such as $\beta$-galactosidase (15). The individual reporter enzyme portions are themselves enzymatically inactive, but when they are brought together through the interaction of their fusion partners, enzymatic activity is generated. Thus, the degree of enzyme fragment complementation acts as an indirect monitor for the degree of protein-protein interaction. A major advantage of FRET, BRET, and PCA strategies is the ability to assess protein interaction dynamics in living cells and in real time. The major drawback to these methods, however, is the need to add large fusion tags to proteins of interest, thereby increasing the chance of altered protein function and/or localization. In contrast, epitope-tagging PLA affords the advantage of fusing proteins of interest to relatively inert epitope tag peptides instead of large, bulky fluorophores/reporters. Furthermore, the ability to investigate protein interactions at the single-molecule level is an attractive feature to this strategy. Another practical benefit of this approach is the possibility of altering the proximity distance limits for interaction studies by changing the lengths of oligonucleotide extensions on the secondary probes, thereby allowing the PLA strategy to act as a "molecular ruler" for defining interaction distances. The downside to our approach is the need for sample fixation and permeabilization, which precludes the prospect of live-cell interaction analysis.

Based on the dramatic success of Herceptin (Genentech USA, San Francisco, CA, USA) in the treatment of ErbB2-overexpressing metastatic breast cancers (16), there is increasing interest in design and testing of rational therapeutics toward EGFR and ErbB member activity (17,18), with clinical trials in progress for the anti-Erb2 antibody Pertuzumab (19) and the EGFR dimerization inhibitor
Matuzumab (20). This in situ PLA EGFR dimerization method may be valuable in the development and testing of additional candidate EGFR interaction inhibitors. Future studies involving application of in situ PLA for EGFR dimerization analysis have the potential to illuminate our understanding of EGFR interactions among mutant EGFR variants as well as distinguish previously uncharacterized interactions with other RTKs.

\section{Acknowled giments}

We gratefully acknowledge Erik Nyström for helpful discussions and technical assistance, and Jane McGlade and Avi Chakrabartty for critical review of the manuscript. This work was supported in part by the Hospital for Sick Children Foundation Student Scholarship program-RESTRACOMP (to A. Gajadhar) and funds from the National Cancer Institute of Canada (to A. Guha).

\section{Competing interests}

The authors declare no competing interests.

\section{References}

1. Hynes, N.E. and H.A. Lane. 2005. ERBB receptors and cancer: the complexity of targeted inhibitors. Nature Reviews 5:341-354.

2. Yarden, Y. and J. Schlessinger. 1987. Epidermal growth factor induces rapid, reversible aggregation of the purified epidermal growth factor receptor. Biochemistry 26:1443-1451.
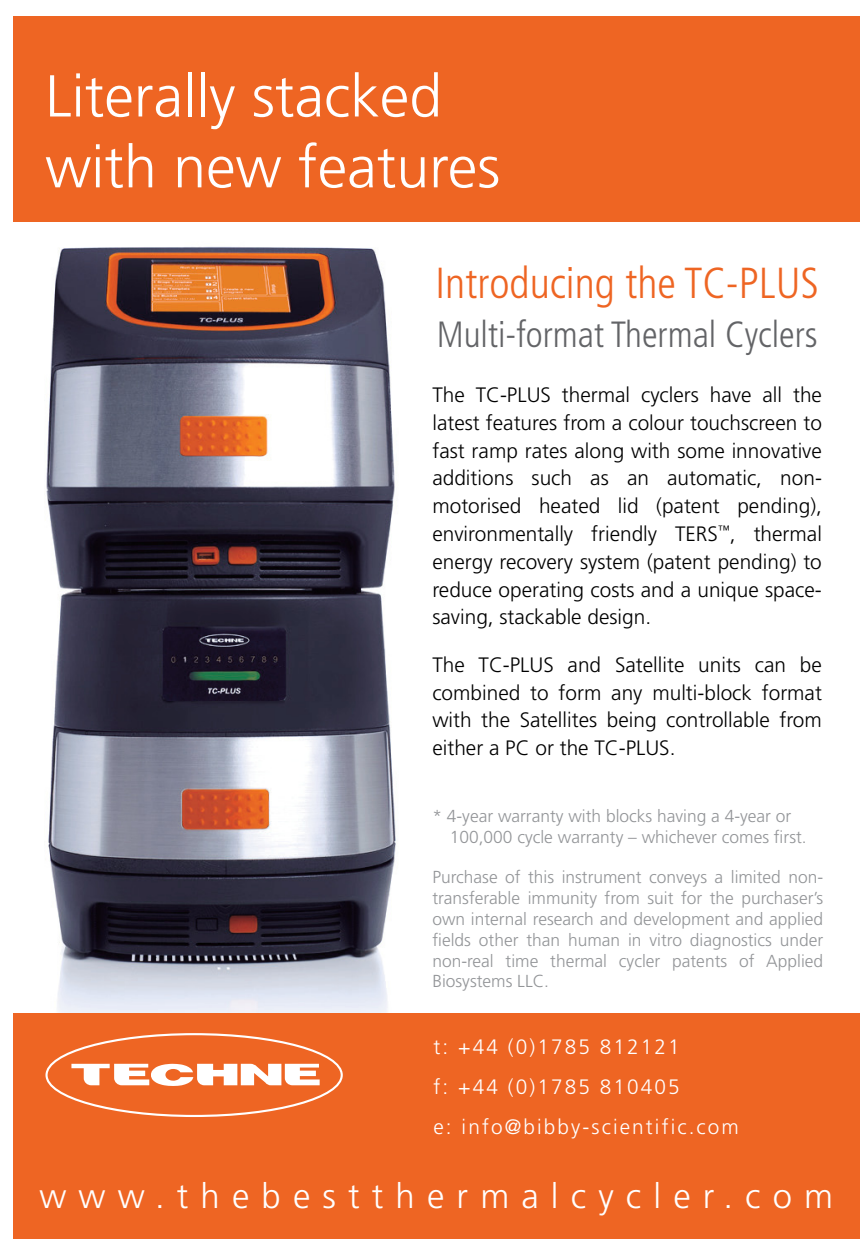
3. Yarden, Y. and J. Schlessinger. 1987. Selfphosphorylation of epidermal growth factor receptor: evidence for a model of intermolecular allosteric activation. Biochemistry 26:1434-1442.

4.Fredriksson, S., M. Gullberg, J. Jarvius, C. Olsson, K. Pietras, S.M. Gustafsdottir, A. Ostman, and U. Landegren. 2002. Protein detection using proximity-dependent DNA ligation assays. Nat. Biotechnol. 20:473477.

5. Soderberg, O., M. Gullberg, M. Jarvius, K. Ridderstrale, K.J. Leuchowius, J. Jarvius, K. Wester, P. Hydbring, et al. 2006. Direct observation of individual endogenous protein complexes in situ by proximity ligation. Nat. Methods 3:995-1000.

6. Jarvius, M., J. Paulsson, I. Weibrecht, K.J. Leuchowius, A.C. Andersson, C. Wahlby, M. Gullberg, J. Botling, et al. 2007. In situ detection of phosphorylated platelet-derived growth factor receptor beta using a generalized proximity ligation method. Mol. Cell. Proteomics 6:1500-1509.

7. Downward, J., P. Parker, and M.D. Waterfield. 1984. Autophosphorylation sites on the epidermal growth factor receptor. Nature 311:483-485.

8. Garrett, T.P., N.M. McKern, M. Lou, T.C. Elleman, T.E. Adams, G.O. Lovrecz, H.J.Zhu, F. Walker, et al. 2002. Crystal structure of a truncated epidermal growth factor receptor extracellular domain bound to transforming growth factor alpha. Cell 110:763-773.
9. Walker, F., S.G. Orchard, R.N. Jorissen, N.E. Hall, H.H. Zhang, P.A. Hoyne, T.E. Adams, T.G. Johns, et al. 2004. CR1/CR2 interactions modulate the functions of the cell surface epidermal growth factor receptor. J. Biol. Chem. 279:22387-22398.

10. Moriki, T., H. Maruyama, and I.N. Maruyama. 2001. Activation of preformed EGF receptor dimers by ligand-induced rotation of the transmembrane domain. J. Mol. Biol. 311:1011-1026.

11. Yu, X., K.D. Sharma, T. Takahashi, R. Iwamoto, and E. Mekada. 2002. Ligandindependent dimer formation of epidermal growth factor receptor (EGFR) is a step separable from ligand-induced EGFR signaling. Mol. Biol. Cell 13:2547-2557.

12.Sako, Y., S. Minoghchi, and T. Yanagida. 2000. Single-molecule imaging of EGFR signalling on the surface of living cells. Nat. Cell Biol. 2:168-172.

13. Selvin, P.R. 2000. The renaissance of fluorescence resonance energy transfer. Nat. Struct. Biol. 7:730-734.

14.Pfleger, K.D. and K.A. Eidne. 2006. Illuminating insights into protein-protein interactions using bioluminescence resonance energy transfer (BRET). Nat. Methods 3:165174

15. Rossi, F., C.A. Charlton, and H.M. Blau. 1997. Monitoring protein-protein interactions in intact eukaryotic cells by beta-galactosidase complementation. Proc. Natl. Acad. Sci. USA 94:8405-8410.
16. Hall, P.S, and D.A. Cameron. 2009 Current perspective-trastuzumab. Eur. J. Cancer 45:12-18.

17. Schmitz, K.R. and K.M. Ferguson. 2009 Interaction of antibodies with ErbB receptor extracellular regions. Exp. Cell Res. 315:659670.

18. Burgess, A.W. 2008. EGFR family: structure physiology signalling and therapeutic targets. Growth Factors 26:263-274.

19. Franklin, M.C., K.D. Carey, F.F Vajdos, D.J. Leahy, A.M. de Vos, and M.X. Sliwkowski. 2004. Insights into ErbB signaling from the structure of the ErbB2-pertuzumab complex. Cancer Cell 5:317-328.

20.Seiden, M.V., H.A. Burris, U. Matulonis, J.B. Hall, D.K. Armstrong, J. Speyer, J.D. Weber, and F. Muggia. 2007. A phase II trial of EMD72000 (matuzumab), a humanized anti-EGFR monoclonal antibody, in patients with platinum-resistant ovarian and primary peritoneal malignancies. Gynecol. Oncol. 104:727-731

Received 24 July 2009; accepted 14 December 2009.

Address correspondence to Abhijit Guha, The Hospital for Sick Children, MaRS-TMDT, Room 11-401E, 101 College St., Toronto, Ontario, Canada, M5G 1L7. e-mail: Abhijit. Guha@uhn.on.ca

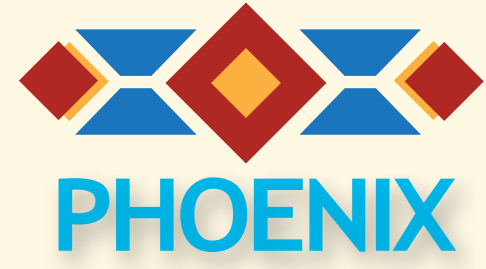

\section{April 11-15, 2010}

Phoenix Convention Center

Phoenix, Arizona, USA

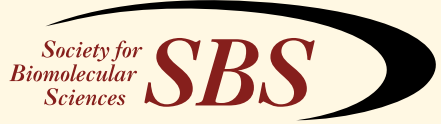

Society for Biomolecular Sciences 36 Tamarack Avenue, \#348, Danbury, CT 06811, USA
SBS 16th Annual Conference \& Exhibition Advancing the Science of Drug Discovery

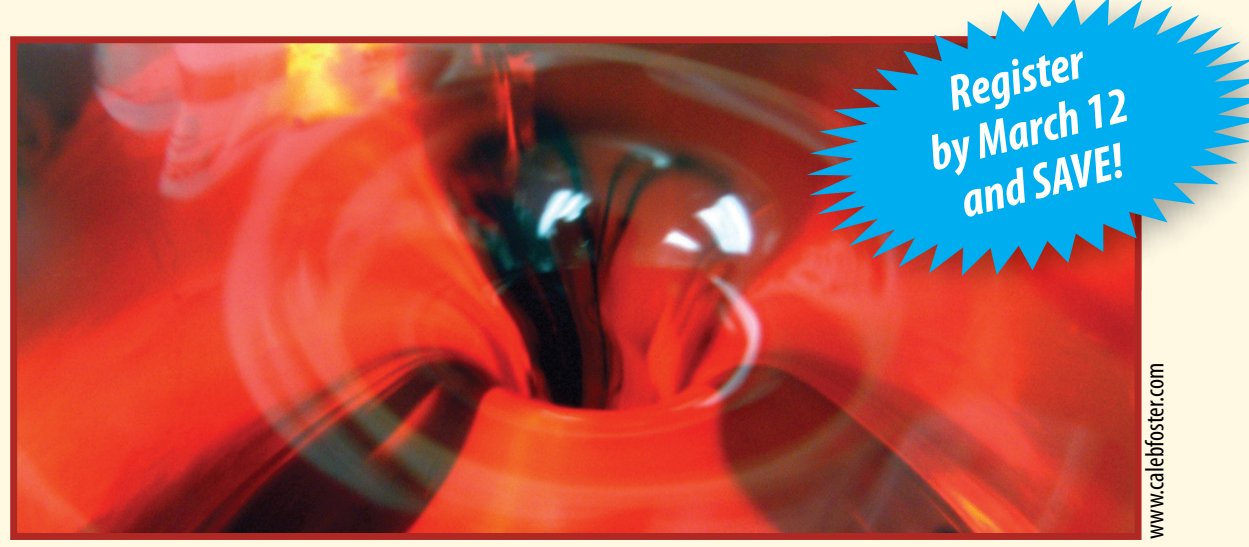

During this five-day event, more than 2,000 scientists, innovators, researchers and industry analysts from around the world will converge in Phoenix to learn about the latest trends and basic and applied research that are transforming the way new pharmaceuticals are developed. 